\title{
ARTICLE
}

\section{lonization-induced annealing of pre-existing defects in silicon carbide}

\author{
Yanwen Zhang ${ }^{1,2}$, Ritesh Sachan1, Olli H. Pakarinen1,3, Matthew F. Chisholm¹, Peng Liu²,4, Haizhou Xue ${ }^{2}$ \\ \& William J. Weber ${ }^{1,2}$
}

\begin{abstract}
A long-standing objective in materials research is to effectively heal fabrication defects or to remove pre-existing or environmentally induced damage in materials. Silicon carbide ( $\mathrm{SiC}$ ) is a fascinating wide-band gap semiconductor for high-temperature, high-power and high-frequency applications. Its high corrosion and radiation resistance makes it a key refractory/structural material with great potential for extremely harsh radiation environments. Here we show that the energy transferred to the electron system of $\mathrm{SiC}$ by energetic ions via inelastic ionization can effectively anneal pre-existing defects and restore the structural order. The threshold determined for this recovery process reveals that it can be activated by 750 and $850 \mathrm{keV}$ Si and $\mathrm{C}$ self-ions, respectively. The results conveyed here can contribute to SiC-based device fabrication by providing a room-temperature approach to repair atomic lattice structures, and to $\mathrm{SiC}$ performance prediction as either a functional material for device applications or a structural material for high-radiation environments.
\end{abstract}

\footnotetext{
${ }^{1}$ Materials Science and Technology Division, Oak Ridge National Laboratory, Oak Ridge, Tennessee 37831, USA. ${ }^{2}$ Department of Materials Science and Engineering, University of Tennessee, Knoxville, Tennessee 37996, USA. ${ }^{3}$ Department of Physics, University of Helsinki, Helsinki FI-00014, Finland. ${ }^{4}$ School of Physics, Key Laboratory of Particle Physics and Particle Irradiation (MOE), Shandong University, Jinan 250100, China. Correspondence and requests for materials should be addressed to Y.Z. (email: zhangy1@ornl.gov) or to W.J.W. (email: wjweber@utk.edu).
} 
Sim ilicon carbide $(\mathrm{SiC})$ is a wide-band gap semiconductor ${ }^{1-5}$, key refractory ceramic $^{6,7}$ and radiation-tolerant structural material ${ }^{8-11}$ that can be functionalized by ion-implantation doping and has great potential for device and structural applications in space and nuclear radiation environments. The capability to retain an ordered atomic structure is essential for reliable device function or material performance in a radiation environment. In these applications, various defects are induced in $\mathrm{SiC}$ by interactions with electrons ${ }^{12,13}$, ions ${ }^{14-16}$, neutrons ${ }^{17}$ and cosmic rays. During a particle-solid interaction, two distinct energy transfer processes occur: atomic collision cascades and electronic excitation on the atomic and electronic structures, respectively. Often overlooked is the fact that a substantial amount of energy is transferred to electrons directly from such energetic particles or through primary knock-on atoms (PKAs), and this energy can profoundly affect atomic defect evolution. Understanding the effects of electronic energy deposition is particularly important in manufacturing devices, engineering nanoscale structures and predicting material performance for nuclear reactors or space applications where electronic energy deposition and displacement damage occur simultaneously.

In an ordered crystalline structure, the exchange of energy between electrons and atoms, via electron-phonon coupling, leads to local heating. Except for the case of swift heavy ions (see Supplementary Discussion for details), this heating has been either (1) simply neglected in the past under an assumption that the thermal effect is separated from the atomic processes and is dissipated without impact on the atomic structure or (2) more recently considered to enhance defect/damage production (a synergetic effect) ${ }^{18}$, create additional atomic defects (an additive effect) ${ }^{19}$ or cause defect/damage recovery (a competitive effect, induced by swift heavy ion irradiation) ${ }^{20}$. In a defective structure, which is normally the case in many applications, the effects of ionization due to electronic energy loss are largely unknown. Our hypothesis is that ionization effects due to the energy loss to target electrons can anneal pre-existing defects, and therefore may effectively modify or alter microstructure evolution. In the current study, we confirmed this hypothesis and bridged the knowledge gap by quantitatively investigating the ionization effects on pre-existing damage in $\mathrm{SiC}$. A surprisingly low threshold of this recovery process at $\sim 1.4 \mathrm{keV} \mathrm{nm}^{-1}$ induced by $\mathrm{MeV}$ ions is determined, which has significant implications for material performance evaluation in extreme radiation environments.

Radiation effects in $\mathrm{SiC}$ have been extensively investigated. For ion-solid interactions in $\mathrm{SiC}$ at very low energies of up to a few hundred $\mathrm{keV}$ (with an electronic energy loss of $<1 \mathrm{keV} \mathrm{nm}^{-1}$ ), damage is almost solely attributed to energy transfer to the atomic structure, which results in target atoms being directly displaced from their lattice sites and defects being produced via atomic collision cascades ${ }^{15,21,22}$. In the high-energy region often referred as swift heavy ions (such as $870 \mathrm{MeV} \mathrm{Pb}$ ions with an electronic energy loss of $33 \mathrm{keV} \mathrm{nm}^{-1}$ ), the ion energy is solely deposited to the loosely bound electrons and then, through electron-phonon coupling, transferred into atomic motion. Such ionization processes in materials can either anneal pre-existing damage or induce crystalline-to-amorphous and order-disordered tranformations ${ }^{20,23,24}$. Most $\mathrm{SiC}$ applications utilize ions in the intermediate regime where electronic and nuclear energy losses are both significant ${ }^{21,25}$. Examples include: ion implantation, ion beam modification and defect engineering by research institutions and industry; ion beam analytical techniques; and ion simulation to mimic ion and neutron radiation effects in nuclear environments. Limited understanding of the coupled effect on the atomic response of $\mathrm{SiC}$ to the two energy deposition pathways is a long-standing roadblock to full utilization of this functional material. Although some defects in $\mathrm{SiC}$ can be removed by thermal annealing at temperatures below $1,000 \mathrm{~K}$, with recrystallization occurring at much higher temperatures, such as $\sim 1,773 \mathrm{~K}$ (refs 9,26), low-temperature manufacturing steps are essential for restoring crystalline order in device applications at nanometre scales.

Previous studies ${ }^{24,27}$ have demonstrated that swift heavy ion irradiation with electronic energy deposition ranging from 10 to $33 \mathrm{keV} \mathrm{nm}^{-1}$ leads to some damage annealing. Here we report a significant competitive effect, promoted by the electronic energy loss of ions with energies in the intermediate regime accessible to industrial accelerators, whereby nearly complete defect annihilation or damage recovery in pre-damaged $4 \mathrm{H}-\mathrm{SiC}$ is achievable. The ionization-induced annealing process (recovery of the ordered atomic structure) in $\mathrm{SiC}$ has a significant impact on low-temperature processes for eliminating defect production during ion-implantation doping, suppression of single-event upset damage in $\mathrm{SiC}$ devices, enhanced radiation tolerance and reliable performance prediction for materials in extreme radiation environments.

\section{Results}

Displacement damage. To better understand and quantify ionization effects on damage recovery, pre-damaged states were introduced using low-energy ion irradiation with $900 \mathrm{keV} \mathrm{Si}^{+}$ self-ions (see Supplementary Methods-Ion Energy Deposition). The high nuclear stopping power $\left(0.38 \mathrm{keV} \mathrm{nm}^{-1}\right)$ and comparable electronic to nuclear ratio (1.75), in comparison to other ions (Table 1), are responsible for the displacement damage production. Different fractional disorder levels were produced under different fluences, with peak disorder at a depth of $\sim 650 \mathrm{~nm}, S_{0}$, of $0.36,0.72$, and close to 1.0 (the fully amorphous state). Examples are shown in Fig. 1 for the cases of 0.72 and close to 1.0. The sequential evaluation of ionization-induced recovery on these pre-damaged disordered states was carried out at room temperature over a range of electronic energy losses from 1.9 to $7.2 \mathrm{keV} \mathrm{nm}^{-1}$ (Table 1). Such irradiation conditions allow a controlled investigation to separately evaluate the ionization effects without introducing significant displacement damage through elastic collisions and to determine the possible threshold of electronic energy loss for the competitive (ionization-induced self-annealing) effects.

Table 1 | lon irradiation condition and predicted stopping powers using the stopping and range of ions in matter (SRIM) code.

\begin{tabular}{|c|c|c|c|c|c|c|c|}
\hline Ions & Energy & $\mathrm{dE} / \mathrm{d} \mathrm{x}_{\mathrm{ele}^{-}} \mathrm{s}$ & $\mathrm{dE} / \mathrm{d} \mathrm{x}_{\mathrm{Nucl}^{-}}-\mathrm{s}$ & Ratio-s & $\mathrm{dE} / \mathrm{d} \mathrm{x}_{\mathrm{ele}}-\mathrm{p}$ & $\mathrm{dE} / \mathrm{d} \mathrm{x}_{\mathrm{Nucl}}{ }^{-p}$ & Ratio-p \\
\hline $\mathrm{Ni}$ & 21 & 8.2 & $6.6 \times 10^{-2}$ & 124 & 7.6 & $7.9 \times 10^{-2}$ & 96 \\
\hline $\mathrm{Si}$ & 21 & 5.0 & $1.0 \times 10^{-2}$ & 483 & 5.0 & $1.2 \times 10^{-2}$ & 422 \\
\hline O & 6.5 & 2.6 & $6.0 \times 10^{-3}$ & 431 & 2.6 & $7.8 \times 10^{-3}$ & 334 \\
\hline C & 4.5 & 1.8 & $3.7 \times 10^{-3}$ & 496 & 1.8 & $4.7 \times 10^{-3}$ & 383 \\
\hline $\mathrm{Si}$ & 0.9 & 1.6 & $1.2 \times 10^{-1}$ & 13 & 0.65 & $3.8 \times 10^{-1}$ & 1.75 \\
\hline
\end{tabular}

Medium mass ions, their energy $(\mathrm{MeV})$, electronic stopping powers $\mathrm{d} E / \mathrm{dx} x_{\text {ele }}\left(\mathrm{keV} \mathrm{nm}^{-1}\right)$ and nuclear stopping powers $\mathrm{dE} / \mathrm{d} x_{\text {Nucl }}\left(\mathrm{keV} \mathrm{nm}^{-1}\right)$ at both sample surface $(-\mathrm{s})$ and $650 \mathrm{~nm}$, where the damage peak (-p) is produced from the $900 \mathrm{keV} \mathrm{Si}{ }^{+}$irradiation are summarized. The corresponding ratio of $\mathrm{dE} / \mathrm{d} x_{\text {ele }}$ to $\mathrm{dE} / \mathrm{d} x_{\text {Nucl }}$ is also calculated at the surface and the damage peak. 
Ionization-induced annealing. Ionization-induced recovery process in $\mathrm{SiC}$ (see Supplementary Methods-Ion Energy Deposition) is observed at irradiation conditions as low as 4.5 MeV C, with $21 \mathrm{MeV} \mathrm{Ni}$ being the most effective ion beam. Two examples of the annealing effect under $21 \mathrm{MeV} \mathrm{Ni}^{+}$ irradiation are shown in Fig. 1. High-disorder profiles of $\sim 0.72$ and 1.00 are produced using $900 \mathrm{keV} \mathrm{Si}^{+}$at fluences of 6.3 and 12 ions per $\mathrm{nm}^{2}$, respectively. For $21 \mathrm{MeV} \mathrm{Ni}$ the electronic stopping power $\left(S_{\mathrm{e}}\right)$ is $\sim 7$ to $8 \mathrm{keV} \mathrm{nm}^{-1}$ from the surface to the damage peak region, and the nuclear stopping $\left(S_{n}\right)$ is negligible at levels below $\sim 0.1 \mathrm{keV} \mathrm{nm}^{-1}$, within the first micrometre from the surface (Supplementary Fig. 1). Given the low nuclear stopping values and high $S_{\mathrm{e}} / S_{\mathrm{n}}$ ratio (Table 1 ), negligible damage buildup from the nuclear energy deposition within $1 \mu \mathrm{m}$ of the surface region is expected. This assumption is confirmed as no damage buildup is observed on either the $\mathrm{Si}$ or the $\mathrm{C}$ sublattice along the $<0001>$ channelling direction in a pristine crystal under $21 \mathrm{MeV} \mathrm{Ni}^{+}$irradiation for ion fluences of up to 10 ions per $\mathrm{nm}^{2}$ (Supplementary Fig. 2). Significant damage annealing is observed in the pre-damaged region as a result of sequential $\mathrm{Ni}$ irradiations for ion fluences up to 10 ions per $\mathrm{nm}^{2}$ (Supplementary Fig. 3). As shown in Fig. 1a,b, clear damage reduction over the entire damage profile is evident after the lowest-fluence irradiation to 0.2 ion per $\mathrm{nm}^{2}$. The relative recovery rate depends on the initial disorder level, and a relatively larger annealing effect is observed for the less-damaged sample $\left(S_{0}=0.72\right)$. For this highly disordered sample (Fig. 1a), considerable damage recovery is observed after $\mathrm{Ni}$ irradiation at ion fluences up to 3 ions per $\mathrm{nm}^{2}$, and the relative disorder decreases from 0.72 to 0.16 . At an ion fluence of 10 ions per $\mathrm{nm}^{2}$, the $\mathrm{Si}$ ion-induced damage is almost fully healed; and the ordered atomic structure is confirmed, as shown by the very low disorder level. For the high-disorder sample with $S_{0}=1.0$, a higher-fluence $\mathrm{Ni}$ beam is needed to repair the pre-existing damage, as shown in

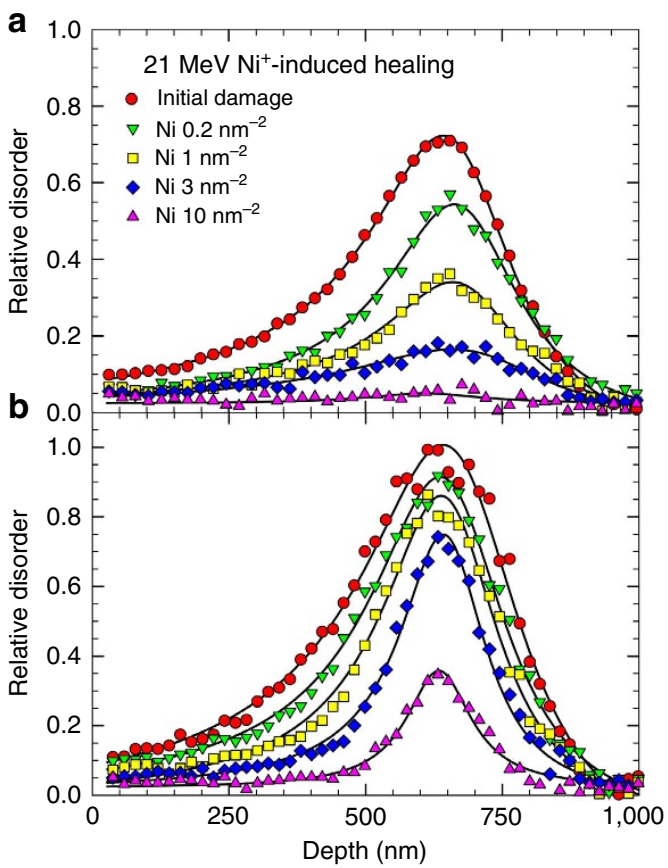

Figure 1 | Damage recovery in SiC under $21 \mathrm{MeV} \mathrm{Ni}^{+}$ion irradiations. The initial damage states of (a) 0.72 and (b) 1.0 were produced by $900 \mathrm{keV}$ $\mathrm{Si}^{+}$with a fluence of 6.3 and 12 ions per $\mathrm{nm}^{2}$, respectively. Reduction of relative disorder is clearly evident with the increase of $\mathrm{Ni}$ fluence from 0.2 to 10 ions per $\mathrm{nm}^{2}$. The combined statistical and measurement uncertainty is represented by the scattered data points from the fitted lines.
Fig. 1b. Substantial recovery is observed under the Ni irradiations when the ion fluence increases from 3 to 10 ions per $\mathrm{nm}^{2}$, with the disorder levels dropping to 0.75 and 0.36 , respectively. Additional $\mathrm{Ni}$ irradiation is required to fully heal the damaged crystalline structure.

Ionization-induced recovery under $\mathrm{MeV} \mathrm{C}, \mathrm{O}, \mathrm{Si}$ and $\mathrm{Ni}$ ion irradiation is observed as a reduction in disorder with increasing ion fluence. The damage recovery behaviour in the pre-damaged samples with $S_{0}=0.36$ and 0.72 is shown in Fig. 2 . The recovery from disorder, averaged from both the $\mathrm{Si}$ and the $\mathrm{C}$ sublattices, as a function of ion fluence is shown in Fig. $2 \mathrm{a}$ for $\mathrm{O}, \mathrm{Si}$ and $\mathrm{Ni}$ irradiation of the $S_{0}=0.36$ samples. To demonstrate consistent ionization-induced recovery on both the $\mathrm{Si}$ and $\mathrm{C}$ sublattices, the disorder recovery determined from both sublattices is presented in Fig. $2 \mathrm{~b}$ for the $S_{0}=0.72$ samples. Compared with $\mathrm{C}, \mathrm{O}$ and $\mathrm{Si}$ irradiation, a significant recovery from $\mathrm{Ni}$ ion irradiation is evident in Fig. 2b. In the intermediate $\mathrm{MeV}$ energy regime with a high ratio of electronic to nuclear stopping powers (Table 1), the results suggest that energy deposited to the target electronic system can effectively anneal irradiation damage. In addition, the ionization-induced annealing increases with ion mass and ion energy. Moreover, compared with the results from the ions with lower healing power $(\mathrm{O}$ and $\mathrm{Si})$ in Fig. 2a,b, the relative recovery (when normalized to the pre-existing disorder level) is nearly a factor of two higher for the samples with the lower initial disorder. At lower levels of disorder, there is an increasing fraction of simple defects, while at higher levels of disorder,
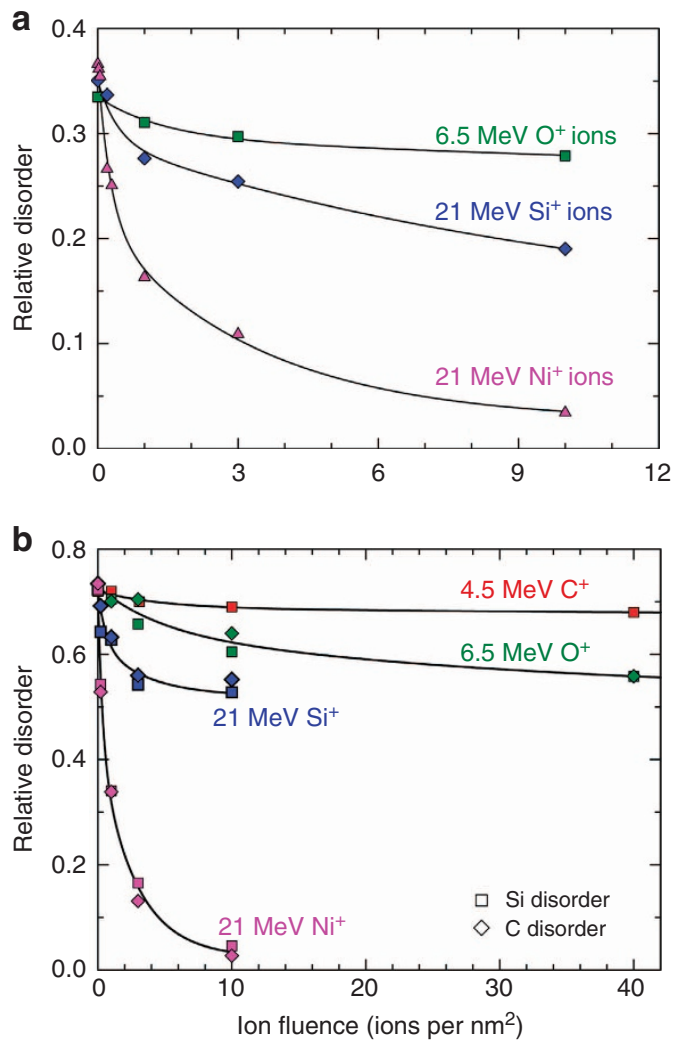

Figure 2 | Ion irradiation-induced healing. Two initial relative disorder levels $S_{O}$ of (a) 0.36 and (b) 0.72 are included as representative. While an average disorder determined from both the $\mathrm{Si}$ and $\mathrm{C}$ sublattices is shown in (a), the actual disorder values on the $\mathrm{Si}$ and $\mathrm{C}$ sublattices are shown in (b). The fluence dependence indicates an effective healing power of $\mathrm{Ni}$ ions, as compared with $\mathrm{Si}, \mathrm{O}$ or $\mathrm{C}$ ions. The measurement uncertainty is $<3 \%$ for disorder determined from the Si sublattice and $<7 \%$ from the $\mathrm{C}$ sublattice. 
more thermally stable defects (for example, clusters and small amorphous domains) are present. Owing to simpler defect types and structure at lower damage levels, recovery is easier in the samples with lower initial disorder.

Under the $\mathrm{MeV}$ irradiation in this study, electronic energy deposition (electronic excitation and ionization) is dominant over atomic energy deposition in the pre-damaged region. Electronic excitation (electronic energy transfer), atomic displacement (momentum transfer) and electron-phonon coupling are entangled with equilibrium heating and non-equilibrium excitation processes ${ }^{21}$. To understand the ionization-induced recovery process at the levels of atoms and electrons, a molecular dynamics approach with a thermal spike model is used. Irradiation-induced recovery from the thermal spike (Supplementary Fig. 4) due to $16 \mathrm{MeV} \mathrm{Ni}$ ions with an electronic energy loss of $\sim 7.2 \mathrm{keV} \mathrm{nm}^{-1}$ was calculated using cubic $24 \times 24 \times 24 \mathrm{~nm}^{3}$ simulation cells $(1.3$ million atoms of $\mathrm{SiC}$ ) containing Frenkel defect concentrations of $\sim 0.1$ and $1.0 \%$ (Fig. 3a). The initial damage is shown as ' 0 ' overlapping ions. Energetic ions lose their energy as they travel through solids. The $\mathrm{Ni}$ energy of $16 \mathrm{MeV}$ was chosen, since this is the average energy of the $21 \mathrm{MeV} \mathrm{Ni}$ ions at the depth of the predamage peak, $\sim 650 \mathrm{~nm}$ below the sample surface. As shown in Fig. 3, a more effective healing effect is observed for the case with a lower defect concentration, consistent with our experimental results shown in Fig. 1. To evaluate the efficiency of the annealing power with different electronic stopping powers, molecular dynamics simulations of $\mathrm{O}$ ion impacts were also performed. The ion energy of $6.5 \mathrm{MeV}$ is chosen for its flat energy deposition profile within the first micrometre of depth in SiC (Supple-
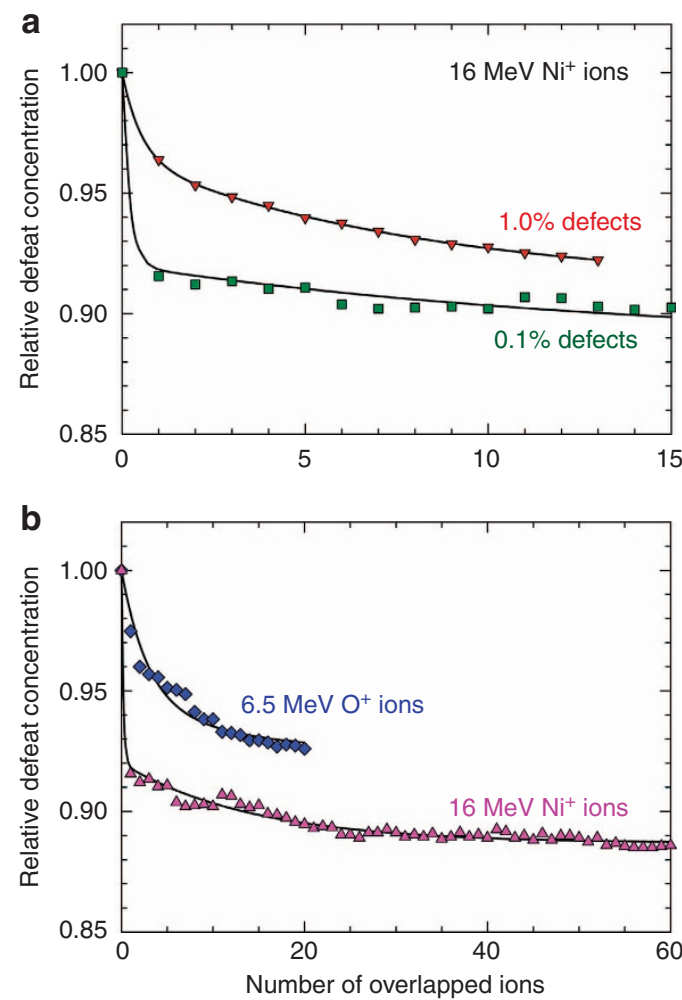

Figure 3 | Molecular dynamics simulation of ionization-induced healing. Decrease of the relative defect concentration is shown as a function of overlap ions: (a) $16 \mathrm{MeV} \mathrm{Ni}$ ions in a simulation cell with initial disorder levels of 1.0 and $0.1 \%$ and (b) $6.5 \mathrm{MeV} \mathrm{O}$ ions and $16 \mathrm{MeV} \mathrm{Ni}$ ions in a simulation cell containing $0.1 \%$ Frenkel pairs, respectively. The initial damage state is marked as ( 0 ) before the first (1) ion. The statistical error is represented by the scattered data points from the fitted lines. mentary Fig. 1). A comparison of the annealing powers resulting from the thermal spike induced by $6.5 \mathrm{MeV} \mathrm{O}$ and $16 \mathrm{MeV} \mathrm{Ni}$ ions on an initial defect concentration of $0.1 \%$ is shown in Fig. $3 b$. Significant recovery attributed to the initial ion impact, which heals the most unstable defects, is observed in both cases, similar to what is shown experimentally in Fig. 2. Furthermore, our molecular dynamics simulations suggest that, on average, about half of the healing (decrease of the number of coordination defects in the cell) from one ion is completed within 4 ps from the ion passage, and a few percent stay until $80-100$ ps. For the initial ion, this annealing process is even faster, $50 \%$ within $1 \mathrm{ps}$, almost $90 \%$ in 10 ps and $\sim 1 \%$ occurring during the $80-100$ ps time frame. A previous molecular dynamics study ${ }^{28}$ using the same interatomic potential has shown that recovery of close Frenkel pairs occurs at activation energies as low as $0.22 \mathrm{eV}$ for several interstitial configurations on sub-picosecond timescales at 2,000 K (supplementary Fig. 4). Similar molecular dynamics studies ${ }^{29}$ of interstitial self-diffusion in $\mathrm{SiC}$ have shown that the transition time for single interstitial hops to be on the order of $0.01 \mathrm{ps}$ at $1,500 \mathrm{~K}$. Moreover, for the same number of overlapping ions, high-energy $\mathrm{Ni}$ ions display a much more effective recovery process, again consistent with the experimental results shown in Fig. 2. It is worth noting that both the simulation and the experimental results suggest damage recovery with an exponential dependence on ion fluence or number of overlapping ions.

Atomic-level microstructure characterization. To validate the annealing effect and confirm defect annihilation, high-resolution microstructural analysis was carried out. The top panel of Fig. 4 shows high-angle annular dark field (HAADF) images of the $4 \mathrm{H}-\mathrm{SiC}$ samples, including a virgin undamaged sample and a predamaged sample with $S_{0}=0.72$ before and after $21 \mathrm{MeV} \mathrm{Ni}{ }^{+}$ irradiation to an ion fluence of 10 ions per $\mathrm{nm}^{2}$. The images were all taken at the same depth $(\sim 500 \mathrm{~nm}$ below surface $)$ with the sample oriented along the $(11 \overline{2} 0)$ zone axis. Compared with the undamaged structure (Fig. 4a), larger variation in the atomic contrast is evident in the $\mathrm{Si}^{+}$-irradiated $\mathrm{SiC}$ (Fig. 4b) as a result of atomic displacement from the irradiation. Surprisingly, the damaged structure is nearly completely healed as a result of irradiation with $21 \mathrm{MeV} \mathrm{Ni}$ ions (Fig. 4c). Along with atomic number, the contrast in the HAADF images also contains information regarding lattice distortion resulting from atomic displacements (see Supplementary Methods-Determination of Atomic Displacements, and Supplementary Fig. 5). The atomic displacements from an ideal position of the Si sublattice in $\mathrm{SiC}$ are mapped in Fig. 4 (lower panel), which gives a fingerprint of the $\mathrm{Si}$ sublattice distortion due to various ion irradiation events corresponding to each atom shown in the top panel. The colour maps in the lower panel represent the vector modulus of the $\mathrm{Si}$ atom displacement (Supplementary Fig. 5). Whereas a high level of contrast variation (large atomic displacement from the ideal lattice position) is evident in the $900 \mathrm{keV} \mathrm{Si}^{+}$pre-damaged $\mathrm{SiC}$ (Fig. 4e), nearly identical contrast levels are observed in the virgin structure (Fig. 4d) and the one annealed by $\mathrm{Ni}$ ion irradiation (Fig. 4f). On the basis of the HAADF images and the detailed atomic displacement analysis, it is quite clear that displacement cascade damage introduced by $900 \mathrm{keV} \mathrm{Si}^{+}$ion irradiation is healed by the electronic energy deposition from the $21 \mathrm{MeV} \mathrm{Ni}$ irradiation.

To quantify the annealing power, irradiation-induced recovery cross-sections, depending on both the initial disorder level and electronic energy loss, are determined (see Supplementary Methods-Determination of Scattering Cross Section). The results in Fig. 5 show the recovery cross-sections for various ions $(\mathrm{C}, \mathrm{O}, \mathrm{Si}$ and $\mathrm{Ni}$ ) with an initial relative disorder of 0.72 or 
a

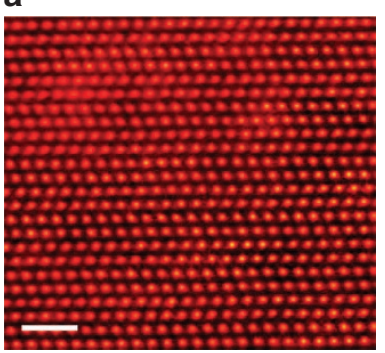

d

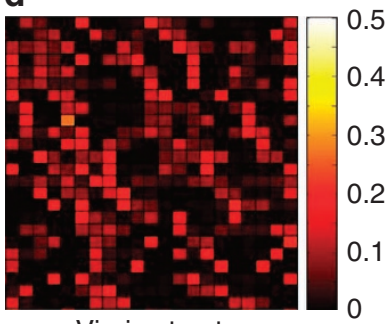

b

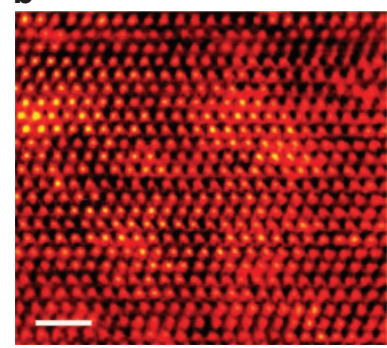

e

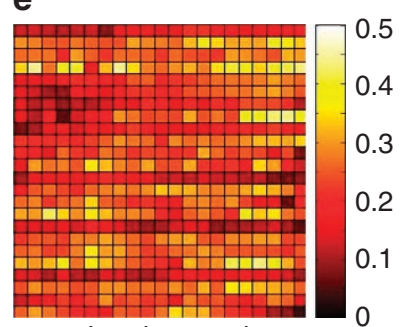

C

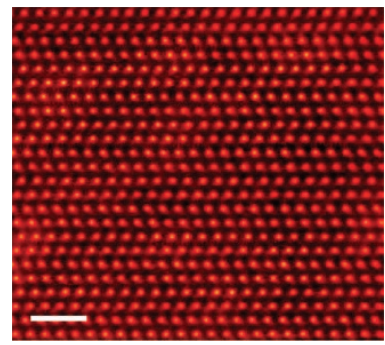

f

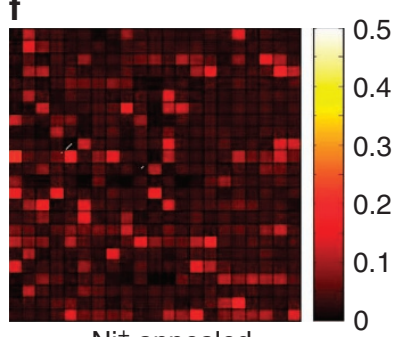

$\mathrm{Ni}^{+}$annealed

Figure 4 | Atomic-level damage recovery. HAADF images (a-c) and the atomic displacement map (d-f) of a virgin (a) and pre-damaged sample $\left(S_{0}=0.72\right)$ before $(\mathbf{b}, \mathbf{e})$ and after $(\mathbf{c}, \mathbf{f}) 21 \mathrm{MeV} \mathrm{Ni}^{+}$irradiation to 10 ions per $\mathrm{nm}^{2}$. The scale bars on the HAADF images correspond to $1 \mathrm{~nm}$. The vector modulus $(\mathbf{d}-\mathbf{f})$ of the corresponding HAADF images $(\mathbf{a}-\mathbf{c})$ represents the displacement of each atom from its ideal position. The colour bar represents the vector modulus with a measurement error bar of $0.1 \AA$.

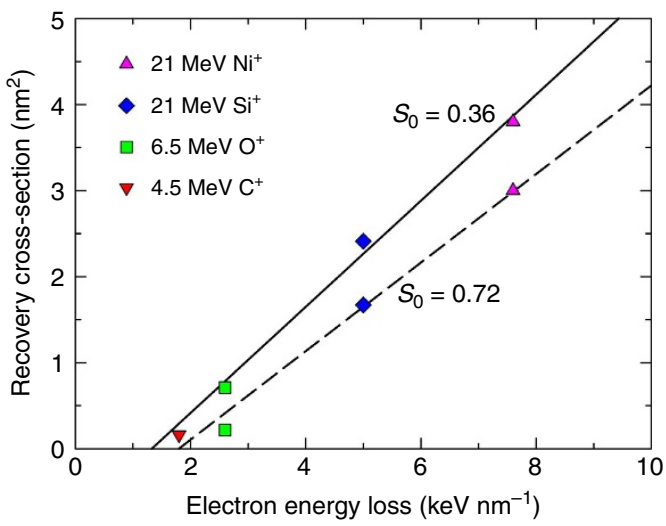

Figure 5 | lonization-induced recovery cross-section. The cross-section is derived from samples with initial disorder levels $S_{0}$ of 0.36 and 0.72 as a function of electronic energy loss. The effective cross-section depends on the ions $(\mathrm{C}, \mathrm{O}, \mathrm{Si}$ and $\mathrm{Ni}$ ) and the initial damage state. The combined measurement and fitting uncertainty is $<10 \%$.

0.36 as a function of electronic energy loss. Significantly increased recovery is observed with increasing electronic energy loss from 1.9 to $7.2 \mathrm{keV} \mathrm{nm}^{-1}$ for ions from $\mathrm{C}$ to $\mathrm{Ni}$ (Table 1) that is attributed to relatively larger recovery cross-sections of $21 \mathrm{MeV}$ $\mathrm{Ni}$ compared with those of $4.5 \mathrm{MeV} \mathrm{C}$. For the lower mass of $\mathrm{O}$ and $\mathrm{C}$ ions, the maximum electronic stopping power (Supplementary Fig. 1) is at $\sim 2.6 \quad(6.5 \mathrm{MeV} \quad \mathrm{O})$ and $1.9 \mathrm{keV} \mathrm{nm}^{-1}$ (4.5 $\left.\mathrm{MeV} \mathrm{C}\right)$; the effective recovery cross-sections are, therefore, limited. The experimental and modelling results (Figs 1, 3 and 5) also indicate that the recovery process depends on the pre-existing defect structure and is more effective at lower initial damage levels or defect concentrations. The effective crosssection, therefore, depends on the initial damage state, which cannot simply be described based solely on the electronic stopping power. A threshold at about $1.4 \mathrm{keV} \mathrm{nm}^{-1}$ is predicted based on a linear fit of the low-disorder state (0.36).

\section{Discussion}

In our combined approach based on ion channelling measurements, atomic-level microstructural analysis and large-scale atomistic simulations, we quantify the effects of electronic energy loss on pre-irradiation-induced lattice damage in SiC. These results provide a scientific understanding of the effects of ionization on ceramics damaged by irradiation. Such electron excitation-induced material modifications should be taken into account for in situ transmission electron microscope studies under ion irradiation, in which simultaneous ionization-induced damage recovery/evolution should be considered. Moreover, our results demonstrate that electronic energy loss from ions and their PKAs can repair damaged SiC lattices at unexpectedly low values of electronic energy deposition, with a threshold value of $1.4 \mathrm{keV} \mathrm{nm}^{-1}$ at room temperature. The threshold PKA energies required to activate these processes in neutron-irradiation environments at room temperature are, therefore, 750 and $850 \mathrm{keV}$ for $\mathrm{Si}$ and $\mathrm{C}$ PKAs, respectively. It is known that $\mathrm{SiC}$, as a key nuclear material for extreme radiation environments ${ }^{30}$, is considered for use as a structural material and a fuel coating in fission reactors ${ }^{9,31}$, for structural components in fusion reactors ${ }^{32}$ and as an inert matrix for transmutation of plutonium and other radioactive waste ${ }^{33,34}$. $\mathrm{SiC}$ is also considered for use as an accident-tolerant cladding for light water reactors and in structural components for advanced high-temperature gascooled reactors. The energies of PKAs created by fusion neutrons and accelerator-based neutron sources, as well as the energies of ions used to investigate neutron damage in materials, are in the intermediate regime where significant ionization effects demonstrated in the current work should not be overlooked.

The significant ionization-induced annealing power can effectively remove nearly all the radiation-induced defects, which is different from the ion beam-enhanced crystallization process that relies on higher temperature to promote atomic mobility where substantial crystalline damage remains. While previous work has focused on high-energy ions and shown ionization effects from swift heavy ions ( $\mathrm{W}, \mathrm{Pb}, \mathrm{Bi}$, etc. $)^{24,27,35,36}$ with relatively high thresholds $\left(>10 \mathrm{keV} \mathrm{nm}^{-1}\right)$, we call attention to 
non-negligible ionization effects from light ions $(\mathrm{C}, \mathrm{O}, \mathrm{Si}$ and $\mathrm{Ni}$ ) with energies of a few MeVs. From separate effect experiments and modelling work, ion annealing at room temperature in the low-energy end of the $\mathrm{MeV}$ range, with a low threshold of electronic energy deposition, is conclusively demonstrated. It is worth noting that a threshold of $>15 \mathrm{keV} \mathrm{nm}^{-1}$ has been reported for defect annealing induced by electronic energy loss over a wider range of pure metals ${ }^{37}$. Owing to different bonding and energy dissipation pathways in metal (metallic bonding) and $\mathrm{SiC}$ (covalent and ionic bonding in ceramics), the difference in threshold values is largely attributed to significant difference in energy deposition profiles between pure metals and $\mathrm{SiC}$, resulting from much higher electronic and thermal conductivities, as well as larger values of electron mean free path in metals. This recovery process with a low threshold $\left(1.4 \mathrm{keV} \mathrm{nm}^{-1}\right)$ may significantly extend the performance lifetime of $\mathrm{SiC}$ in fusion reactor environments. In terms of fundamental research, this ionization-induced annealing power may have a significant impact on prediction of radiation damage accumulation in $\mathrm{SiC}$ and other nuclear materials responding to fast neutrons, accelerator-based neutrons or surrogate ions. Consideration of ionization-induced recovery in $\mathrm{SiC}$ is critical for reliable performance evaluation.

In summary, we have investigated ionization-induced healing of ballistic damage in $\mathrm{SiC}$ and identified an unexpectedly low threshold value of electronic energy loss for initiating the healing process at room temperature. Our findings are validated by a substantial reduction in dechannelling yield due to the effective annihilation of a high concentration of interstitials and small defect clusters over a sub-micrometre depth, by evidence of a repaired crystalline structure with much less observed displacements at the atomic level and by insights into corresponding defect dynamic processes revealed by molecular dynamics simulations. Understanding this recovery mechanism in $\mathrm{SiC}$ has significant implications for the study of irradiation effects in other ceramics for applications in extreme radiation environments. Scientific advances based on this work not only will facilitate the design of radiation-tolerant materials for advanced nuclear energy systems and space exploration but also will contribute to a foundation for the design and control of material properties. That foundation will enable broad advances in device fabrication, sustainable energy technologies and national security involving materials subjected to ion beam modification or severe radiation environments.

\begin{abstract}
Methods
Ion irradiation. Quantitative damage recovery studies were performed by first introducing different disorder levels containing various concentrations of Frenkel pairs and defect clusters in $\mathrm{SiC}$ through ballistic collision processes initiated by low-energy $900 \mathrm{keV} \mathrm{Si}^{+}$ions. The damage profile is peaked at $\sim 650 \mathrm{~nm}$ from the surface. Ionization-induced recovery was quantitatively investigated using high-energy ions of $4.5 \mathrm{MeV} \mathrm{C}, 6.5 \mathrm{MeV} \mathrm{O}, 21 \mathrm{MeV} \mathrm{Si}$ and $21 \mathrm{MeV} \mathrm{Ni}$ at room temperature (see Supplementary Methods-Ion Energy Deposition). The ion flux was $1.7 \times 10^{12} \mathrm{~cm}^{-2} \mathrm{~s}^{-1}$ for $\mathrm{C}$ and $\mathrm{O}, 1.7 \times 10^{11} \mathrm{~cm}^{-2} \mathrm{~s}^{-1}$ for $\mathrm{Si}$ and $1.5 \times 10^{11} \mathrm{~cm}^{-2} \mathrm{~s}^{-1}$ for $\mathrm{Ni}$, respectively. These $\mathrm{MeV}$ ions deposit their energy with very high ratios of electronic to nuclear energy loss (Table 1).
\end{abstract}

Ion beam analysis. Irradiation-induced damage in crystalline samples was quantified using backscattering techniques: Rutherford backscattering spectrometry (RBS) and non-Rutherford backscattering spectrometry (NRBS). Helium ions with an energy of $3.5 \mathrm{MeV}$ were employed to significantly enhance the scattering cross-section of $\mathrm{C}$ atoms, and the disorder analysis on both $\mathrm{Si}$ (RBS) and $\mathrm{C}$ (NRBS) sublattices along the $<0001>$ direction was performed from a single channelling backscattering measurement (see Supplementary Methods-Ion Channeling Measurements). If a crystal contains displaced lattice atoms, there will be an increased yield resulting from direct backscattering and dechannelling of the probing ions due to the interaction with the displaced atoms. Following the $900 \mathrm{keV} \mathrm{Si}^{+}$irradiations, subsequent in situ channelling measurements were carried out, with a Si detector located at a scattering angle of $155^{\circ}$ relative to the incoming beam, before and after the additional $\mathrm{MeV}$ irradiations. The channelling spectra were analysed using an iterative procedure to achieve the relative disorder level $^{25}$.

Molecular dynamics. Large-scale classical molecular dynamics studies were performed that simulated electronic energy loss generated by passing ions ${ }^{38}$. The radial distribution of electronic heating from the passing incident ions (supplementary Fig. 4) is determined by applying the thermal spike model ${ }^{39}$. The local temperature from ionization as a function of time and radial distance from the ion trajectory was calculated and transferred to atoms along the ion track as kinetic energy at the beginning of the molecular dynamics simulation (see Supplementary Methods-Molecular Dynamics Simulations). Irradiation-induced recovery due to a series of directly overlapping $16 \mathrm{MeV}$ Ni ions, each separated by a 120 -ps relaxation to $300 \mathrm{~K}$ temperature, was calculated using simulation cells containing Frenkel defects of both $\sim 0.1$ and $1.0 \%$, as shown in Fig. 3a. The $16-\mathrm{MeV} \mathrm{Ni}$ and $6.5-\mathrm{MeV} \mathrm{O}$ results were compared using a cell with an initial disorder level of $0.1 \%$.

STEM characterization. Samples were prepared for scanning transmission electron microscope (STEM) analysis using a focused ion beam (FIB) in a cross beam Zeiss Auriga FIB/SEM. A lamella of 800-nm thickness was prepared using a gallium FIB beam probe with a current of $2 \mathrm{nA}$ at $30 \mathrm{KV}$. The current was gradually reduced to $10 \mathrm{pA}$ to minimize FIB beam damage while thinning the lamella to $\sim 100 \mathrm{~nm}$. As a final step, a Fischione 1040 nanomill was used to further thin the sample at $900 \mathrm{eV}$ from each side of the sample at an angle of $\pm 7^{\circ}$ for $5 \mathrm{~min}$. HAADF imaging was performed on various samples in a fifth-order aberration-corrected STEM (Nion UltraSTEM200) operating at $200 \mathrm{KV}$. A detector with an inner angle of $65 \mathrm{mrad}$ was used to collect electrons for HAADF imaging. The electron probe current and the exposure time/pixel for imaging in the experiment were $30 \mathrm{pA}$ and $16 \mu \mathrm{s}$, respectively, to minimize the electron beaminduced modification.

\section{References}

1. Madar, R. Materials science: silicon carbide in contention. Nature 430, 974-975 (2004).

2. Lee, T-H., Bhunia, S. \& Mehregany, M. Electromechanical computing at $500^{\circ} \mathrm{C}$ with silicon carbide. Science 10, 1316-1318 (2010).

3. Obermayer, D., Gutmann, B. \& Kappe, C. O. Microwave chemistry in silicon carbide reaction vials: separating thermal from nonthermal effects. Angew. Chem. Int. Ed. 48, 8321-8324 (2009).

4. Eddy, Jr. C. R. \& Gaskill, D. K. Silicon carbide as a platform for power electronics. Science 324, 1398-1400 (2009).

5. Nakamura, D. et al. Ultrahigh-quality silicon carbide single crystals. Nature 430, 1009-1012 (2004).

6. Tredway, W. K. Toughened ceramics. Science 282, 1275-1275 (1998).

7. Ishikawa, T. et al. A tough, thermally conductive silicon carbide composite with high strength up to $1600^{\circ} \mathrm{C}$ in air. Science 282, 1295-1297 (1998).

8. Ochedowski, O. et al. Graphitic nanostripes in silicon carbide surfaces created by swift heavy ion irradiation. Nat. Commun. 5, 3913 (2014).

9. Jones, R. H. et al. Promise and challenges of $\mathrm{SiCf} / \mathrm{SiC}$ composites for fusion energy applications. J. Nucl. Mater. 307-311, 1057-1072 (2002).

10. Snead, L. L. et al. Handbook of SiC properties for fuel performance modeling J. Nucl. Mater. 371, 329-377 (2007).

11. Zhang, Y. et al. Nanoscale engineering of radiation tolerant silicon carbide. Phys. Chem. Chem. Phys. 14, 13429-13436 (2012).

12. Ishimaru, M., Zhang, Y., Shannon, S. \& Weber, W. J. Origin of radiation tolerance in 3C-SiC with nanolayered planar defects. Appl. Phys. Lett. 103, 033104 (2013).

13. Inui, H., Mori, H., Suzuki, T. \& Fujita, H. Electron-irradiation-induced crystalline-to-amorphous transition in $\beta$-SiC single crystals. Phil. Mag. B 65, 1-14 (1992).

14. Jin, K. et al. Electronic stopping powers for heavy ions in $\mathrm{SiC}$ and $\mathrm{SiO}_{2}$. J. Appl. Phys. 115, 044903 (2014)

15. Zhang, Y., Gao, F., Jiang, W., McCready, D. E. \& Weber, W. J. Damage accumulation and defect relaxation in 4H-SiC. Phys. Rev. B 70, 125203 (2004)

16. Jiang, W. et al. Response of nanocrystalline 3C silicon carbide to heavy-ion irradiation. Phys. Rev. B 80, 161301(R) (2009).

17. Snead, L. L. \& Hay, J. C. Neutron irradiation induced amorphization of silicon carbide. J. Nucl. Mater. 273, 213-220 (1999).

18. Toulemonde, M. et al. Synergy of nuclear and electronic energy losses in ion-irradiation processes: the case of vitreous silicon dioxide. Phys. Rev. B 83, 054106 (2011).

19. Zhang, Y. et al. The effect of electronic energy loss on irradiation-induced grain growth in nanocrystalline oxides. Phys. Chem. Chem. Phys. 16, 8051-8059 (2014).

20. Debelle, A. et al. Combined experimental and computational study of the recrystallization process induced by electronic interactions of swift heavy ions with silicon carbide crystals. Phys. Rev. B 86, 100102 (2012). 
21. Zhang, Y., Weber, W. J., Jiang, W., Hallén, A. \& Possnert, G. Damage evolution and recovery on both $\mathrm{Si}$ and $\mathrm{C}$ sublattices in $\mathrm{Al}$-implanted $4 \mathrm{H}-\mathrm{SiC}$ studied by RBS and NRA. J. Appl. Phys. 91, 6388 (2002).

22. Weber, W. J., Duffy, D. M., Thomé, L. \& Zhang, Y. The role of electronic energy loss in ion beam modification of materials. Curr. Opin. Solid State Mater. Sci. 19, 1-11 (2015).

23. Benyagoub, A. \& Audren, A. Mechanism of the swift heavy ion induced epitaxial recrystallization in predamaged silicon carbide. J. Appl. Phys. 106, 083516 (2009)

24. Benyagoub, A., Audren, A., Thomé, L. \& Garrido, F. Athermal crystallization induced by electronic excitations in ion-irradiated silicon carbide. Appl. Phys. Lett. 89, 241914 (2006).

25. Zhang, Y., Debelle, A., Boulle, A., Kluth, P. \& Tuomisto, F. Advanced techniques for characterization of ion beam modified materials. Curr. Opin. Solid State Mater. Sci. 19, 19-28 (2015).

26. Schmidt, H. et al. Crystallization kinetics of amorphous SiC films: influence of substrate. Appl. Surf. Sci. 252, 1460-1470 (2005).

27. Thomé, L. et al. Combined effects of nuclear and electronic energy losses in solids irradiated with dual-ion beam. Appl. Phys. Lett. 102, 141906 (2013).

28. Gao, F. \& Weber, W. J. Recovery of close Frenkel pairs produced by low energy recoils in SiC. J. Appl. Phys. 94, 4348 (2003).

29. Gao, F. et al. Atomistic study of intrinsic defect migration in 3C-SiC. Phys. Rev. B 69, 245205 (2004).

30. Snead, L. L. et al. Stability of SiC-matrix microencapsulated fuel constituents at relevant LWR conditions. J. Nucl. Mater. 448, 389-398 (2014).

31. Xiao, H. et al. Near-surface and bulk behavior of Ag in SiC. J. Nucl. Mater. 420, 123-130 (2012).

32. Zinkle, S. J. \& Busby, J. T. Structural materials for fission and fusion energy. Mater. Today 12, 12-19 (2009).

33. Verrall, R. A., Vlajic, M. D. \& Krstic, V. D. Silicon carbide as an inert-matrix for a thermal reactor fuel. J. Nucl. Mater. 274, 54-60 (1999).

34. Somiya, S. (ed.) Handbook of Advanced Ceramics: Materials, Applications, Processing, and Properties 2nd edn (Elsevier, 2013).

35. Zinkle, S. J., Skuratov, V. A. \& Hoelzer, D. T. On the conflicting roles of ionizing radiation in ceramics. Nucl. Instrum. Methods B 191, 758-766 (2002).

36. Itoh, N., Duffy, D. M., Khakshouri, S. \& Stoneham, A. M. Making tracks: electronic excitation roles in forming swift heavy ion tracks. J. Phys. Cond. Matter 21, 474205 (2009).

37. Wang, Z. G. et al. Defect production and annealing induced by electronic energy loss in pure metal. Nucl. Instrum. Methods B 135, 265-269 (1998).
38. Backman, M. et al. Molecular dynamics simulations of swift heavy ion induced defect recovery in SiC. Comput. Mater. Sci. 67, 261 (2013).

39. Toulemonde, M. et al. Experimental phenomena and thermal spike model description of ion tracks in amorphisable inorganic insulators. Mat. Fys. Medd. Dan. Vid. Selsk 52, 263 (2006).

\section{Acknowledgements}

This work was supported by the U.S. Department of Energy, Office of Science, Basic Energy Sciences, Materials Sciences and Engineering Division. Ion beam work was performed at the University of Tennessee-Oak Ridge National Laboratory Ion Beam Materials Laboratory (IBML) located on the campus of the University of TennesseeKnoxville. The computer simulation used the resources of the National Energy Research Scientific Computing Center, supported by the U.S. Department of Energy Office of Science under Contract No. DEAC02-05CH11231.

\section{Author contributions}

Y.Z. and W.J.W. designed the experiments and performed the data analysis; Y.Z., P.L. and H.X. carried out the ion irradiation and ion channelling measurements; R.S. and M.F.C. carried out the STEM characterization and the quantitative microstructural analysis; O.H.P. performed the molecular dynamics simulations; W.J.W supervised the project; and Y.Z. wrote the manuscript with input from W.J.W., R.S. and O.H.P.

\section{Additional information}

Supplementary Information accompanies this paper at http://www.nature.com/ naturecommunications

Competing financial interests: The authors declare no competing financial interests.

Reprints and permission information is available online at http://npg.nature.com/ reprintsandpermissions/

How to cite this article: Zhang, Y. et al. Ionization-induced annealing of pre-existing defects in silicon carbide. Nat. Commun. 6:8049 doi: 10.1038/ncomms9049 (2015).

(c) (i) This work is licensed under a Creative Commons Attribution 4.0 International License. The images or other third party material in this article are included in the article's Creative Commons license, unless indicated otherwise in the credit line; if the material is not included under the Creative Commons license, users will need to obtain permission from the license holder to reproduce the material. To view a copy of this license, visit http://creativecommons.org/licenses/by/4.0/ 